\title{
Cytosolic phospholipase A2- $\alpha$ expression in breast cancer is associated with EGFR expression and correlates with an adverse prognosis in luminal tumours
}

\author{
F Caiazza', NS McCarthy', L Young', ADK Hill², BJ Harvey' and W Thomas*,I \\ 'Department of Molecular Medicine, Royal College of Surgeons in Ireland, Education and Research Centre, Beaumont Hospital, Dublin 9, Ireland; \\ ${ }^{2}$ Department of Surgery, Royal College of Surgeons in Ireland, Education and Research Centre, Beaumont Hospital, Dublin 9, Ireland
}

BACKGROUND: The eicosanoid signalling pathway promotes the progression of malignancies through the production of proliferative prostaglandins (PGs). Cytosolic phospholipase $\mathrm{A}_{2} \alpha\left(c P L A_{2} \alpha\right)$ activity provides the substrate for cyclooxygenase-dependent $P G$ release, and we have previously found that $c P L A_{2} \alpha$ expression correlated with EGFR/HER2 over-expression in a small number of breast cancer cell lines.

METHODS: The importance of differential $\mathrm{CPLA}_{2} \alpha$ activity in clinical breast cancer was established by relating the expression of $\mathrm{cPLA} \alpha$ in tissue samples from breast cancer patients, and two microarray-based gene expression datasets to different clinicopathological and therapeutic parameters.

RESULTS: High CPLA $2 \alpha$ mRNA expression correlated with clinical parameters of poor prognosis, which are characteristic of highly invasive tumours of the HER2-positive and basal-like subtype, including low oestrogen receptor expression and high EGFR expression. High $\mathrm{CPLA} \mathrm{A}_{2} \alpha$ expression decreased overall survival in patients with luminal cancers, and correlated with a reduced effect of tamoxifen treatment. The $C P L A_{2} \alpha$ expression was an independent predictive parameter of poor response to endocrine therapy in the first 5 years of follow-up.

CONCLUSION: This study shows a role of $\mathrm{CPLA}_{2} \alpha$ in luminal breast cancer progression, in which the enzyme could represent a novel therapeutic target and a predictive marker.

British Journal of Cancer (20I I) I 04, 338-344. doi:I0.1038/sj.bjc.6606025 www.bjcancer.com

Published online 30 November 2010

(c) 201 I Cancer Research UK

Keywords: phospholipase; EGFR; breast cancer; oestrogen

Breast cancer is the most common malignancy diagnosed in women and the leading cause of cancer-related death in women worldwide, accounting for $\sim 500000$ deaths per year and representing $15 \%$ of all female cancer-related mortality (Hortobagyi et al, 2005). Early detection and improved treatment account for the decline in mortality rates that has been observed in the past two decades (Jemal et al, 2007). Molecular characterisation and profiling has improved our understanding of the heterogeneity of breast cancer, and is now an important clinical tool to predict the course of the disease and identify the most appropriate treatment regimen for each patient (Perou et al, 2000; Sorlie et al, 2001; Hu et al, 2006). Accordingly, oestrogen receptor (ER)-positive patients are treated with adjuvant endocrine therapy (such as tamoxifen or fulvestrant; Jordan, 2007), and HER2-positive patients can benefit from targeted agents, such as trastuzumab or lapatinib (Rabindran, 2005). However, the vast majority of patients treated with adjuvant systemic therapy do not respond to the treatment or develop acquired resistance. Furthermore, patients with ER-negative, progesterone receptor (PR)-negative and HER2-negative tumours (triple-negative,

*Correspondence: Dr W Thomas; E-mail: wthomas@rcsi.ie Received II May 20 I0; revised 8 October 2010; accepted 8 November 2010 ; published online 30 November 2010 or basal-like cancers) lack an established therapeutic target and can only be treated with conventional chemotherapy (Linn and Van ' $t$ Veer, 2009). It is, therefore, crucial to improve the translational effort in order to identify molecular markers that could aid in the prediction of tumour progression, prognosis and therapeutic regimen.

The group IVA cytosolic phospholipase $\mathrm{A}_{2}\left(\mathrm{CPLA}_{2} \alpha\right)$ is an enzyme that catalyses the hydrolysis of the $s n-2$ linkage in membrane glycerol-phospholipids to release arachidonic acid (AA), which is then converted to biologically active eicosanoid lipid mediators, including prostaglandin $\mathrm{E}_{2}\left(\mathrm{PGE}_{2}\right)$, produced by cyclooxygenase (COX)-2 (Leslie, 1997). The $\mathrm{PGE}_{2}$ has important regulatory roles in diverse cellular responses, including cell growth and differentiation; accordingly, the AA-based eicosanoid signalling pathway has been implicated in the development and progression of cancer in different human tissues, including the breast (Nakanishi and Rosenberg, 2006; Thomas et al, 2008). Clinical, epidemiological and molecular evidence has linked COX-2 expression/activation and $\mathrm{PGE}_{2}$ production to breast cancer progression (reviewed in Howe, 2007). We have previously reported that $\mathrm{cPLA}_{2} \alpha$ can be rapidly activated by physiological concentrations of $17 \beta$-estradiol (E2) and is involved in the proliferative effects of E2 in breast cancer cell (Thomas et al, 2006, 2008; Caiazza et al, 2010). Specifically, the E2-induced 
activation of $\mathrm{CPLA}_{2} \alpha$ is mediated by trans-activation of EGFR/ HER2 heterodimers signalling through ERK1/2 mitogen-activated protein kinase (Caiazza et al, 2010), resulting in the activation of proliferative signals in both ER-positive and ER-negative breast cancer cells. Pre-clinical data support a role for EGFR/HER2 signalling in promoting E2-independent tumour growth and in the development of resistance to endocrine therapy (Knowlden et al, 2003). In agreement with these data, clinical evidence shows that over-expression of both EGFR and HER2, which is found in $50 \%$ and $30 \%$ of breast cancers, respectively, correlates with a decreased sensitivity to endocrine therapy and with poor patient prognosis (Pietras, 2003). Furthermore, over-expression of HER2 receptors and related signalling intermediates is a molecular determinant of selective loss of ER expression, progression to ER-negative invasive phenotype and development of resistance to selective ER modulator-based therapy (Lopez-Tarruella and Schiff, 2007). Consequently, in the past 20 years, HER2 has become an important prognostic marker and therapeutic target in breast cancer. Taking into account the involvement of EGFR/HER2 receptors in the E2-induced activation of $\mathrm{CPLA}_{2} \alpha$ in breast cancer cell lines, it was hypothesised that $\mathrm{CPLA}_{2} \alpha$ activity and expression could be correlated with HER2-overexpressing tumours, a suggestion that is also supported by previous studies showing a correlation between expression of intermediates in the eicosanoid signalling pathway, particularly COX-2, and HER2 over-expression in breast cancer (Vadlamudi et al, 1999; Ristimaki et al, 2002; Subbaramaiah et al, 2002; Wulfing et al, 2003). We previously reported a novel correlation between $\mathrm{CPLA}_{2} \alpha$ and HER2 over-expression in a small number of cell lines (Caiazza et al, 2010). This study investigates the relationship between $\mathrm{CPLA}_{2} \alpha$ and EGFR/HER2 in vivo, addressing the clinical implications of differential $\mathrm{CPLA}_{2} \alpha$ expression in breast cancer.

\section{PATIENTS AND METHODS}

\section{Patient samples and quantitative real-time PCR (qRT - PCR)}

Ethical approval and patient consent was obtained for access to primary tumour samples from 18 breast cancer patients who underwent surgery at Beaumont Hospital (Dublin, Ireland) between 2008 and 2009. In all, 15 patients were ER positive and 3 were ER negative as determined by standard histopathological evaluation. The patients had not been treated with adjuvant endocrine therapy; however, the majority had received selective oestrogen receptor modulator therapy with tamoxifen. The complete clinicopathological characteristics are summarised in Supplementary Table S1.

RNA extraction and $\mathrm{qRT}-\mathrm{PCR}$ were performed as previously described (Caiazza et al, 2010). Expression levels of HER2 mRNA were normalised to a standard housekeeping gene (18S $r R N A$ ) and calibrated to the MCF-7 cell line chosen to represent $1 \times$ expression of HER2 gene (Barberis et al, 2008) All samples above a threshold of HER2 mRNA expression relative to the calibrator were assigned to the HER2-positive group. This method was previously validated by comparing HER 2 mRNA levels in 55 formalin-fixed, paraffin-embedded samples, in which HER2 status was evaluated by IHC and fluorescence in situ hybridisation (Barberis et al, 2008).

\section{Gene expression microarrays}

The expression of PLA2G4A (probe 210145_at), EGFR (probe 210984_x_at) and ERBB2 (probe 210930_s_at) was examined in two previously published microarray data sets of breast cancer cell lines (Kao et al, 2009) and primary tumour samples (van de Vijver et al, 2002), which had been profiled with an Affymetrix microarray assay (Affymetrix, Santa Clara, CA, USA). Data were downloaded, respectively, from the PLOS website (http://dx.plos. org/10.1371/journal.pone.0006146) and from the original publication on-line supporting information website (http://microarray-pubs. stanford.edu/wound_NKI/explore.html). Comprehensive clinical data of all 295 patients were also available from the same source.

\section{Statistical analysis}

Statistical analysis of the data was performed with paired Student's $t$-test for analysis between two groups. Fisher's exact test was performed on contingency tables to analyse the correlation between two variables. Pearson's coefficient was used to measure correlation between linear variables. Survival curves were calculated according to the Kaplan-Meier method and compared using the Cox-Mantel log-rank test. Overall survival (OS) was calculated as the time between the first date of treatment and the last date of follow-up or the date of death. Relapse-free survival (RFS) was calculated as the time between the first date of treatment and the last date of followup or date of recurrence (loco-regional recurrence or distant metastasis). Kaplan-Meier curves were calculated, as indicated, over the entire follow-up time (17 years) or over a follow-up time of 10 years to focus on the effects on early development of acquired endocrine resistance. All analyses were performed using prism (Graphpad Software, La Jolla, CA, USA). Cox proportional hazard models were computed using XLStat-Life for Microsoft Excel (Kovach Computing Services, Anglesey Wales). The $\mathrm{CPLA}_{2} \alpha$ expression was categorised as high or low using the upper median as a cutoff point. Where indicated, the median was used as a cutoff point to exclude any possible bias because of the low number of patients. All other variables were either dichotomised or analysed as continuous variables as indicated. The number of patients in each group is reported in the figure legend. A heat-map image was generated from microarray data using the Matrix2png web interface (University of British Columbia, http://chibi.ubc.ca/matrix2png; Pavlidis and Noble, 2003). All $P$-values are two-tailed, and $P$-values of $<0.05$ were considered statistically significant.

\section{RESULTS}

\section{Increased expression of $\mathrm{CPLA}_{2} \alpha$ mRNA correlates with HER2 over-expression in breast cancer patients}

The expression of $\mathrm{CPLA}_{2} \alpha$ was analysed by $\mathrm{qRT}-\mathrm{PCR}$ in tumour samples from a cohort of Irish breast cancer patients (complete clinical data for the 18 patients are detailed in Supplementary Table S1). Recently, the validity of IHC to test for HER2 amplification has been questioned in terms of standardisation, reproducibility and accuracy (Gown, 2008), and different groups have reported results supporting a better accuracy of qRT-PCR in detecting HER2 amplification, avoiding false positives and false negatives (Vinatzer et al, 2005; Kulka et al, 2006). Consequently, we quantified HER2 expression in all 18 samples using qRT-PCR. In all, 9 samples out of 18 (50\%) scored as HER2 positive using the described method, including some that were previously classified as HER2 negative using standard pathological evaluation. Expression of $\mathrm{CPLA}_{2} \alpha$ mRNA was significantly $(P<0.01)$ increased in HER2-positive tissue samples (Figure 1).

Increased expression of $\mathrm{cPLA}_{2} \alpha$ mRNA correlates with the basal-like and HER2-positive subtypes, and with markers of poor prognosis in a panel of breast cancer cell lines

With the aim of expanding our study to a larger sample size population, we first analysed the expression of $\mathrm{cPLA}_{2} \alpha$ in a panel of 30 breast cancer cell lines for which gene expression microarray data were previously published (Kao et al, 2009). Increased 
expression of $\mathrm{CPLA}_{2} \alpha$ correlated with high expression of EGFR and with low expression of ER $\alpha$ at the mRNA level (Figure 2A). No significant correlation was found between CPLA $_{2} \alpha$ and HER2 expression, but this was influenced by missing data for HER2 mRNA expression levels. High $\mathrm{cPLA}_{2} \alpha$ abundance clustered in cell lines characterised by basal phenotype compared with luminal phenotype, and cPLA2 $\alpha$ expression also correlated with basal-like and HER2-positive subtypes as opposed to luminal A and luminal $B$ subtypes (Figure 2B). High EGFR expression, low ER $\alpha$, the basal and HER2-positive subtypes are all molecular markers of poor prognosis in breast cancer. High $\mathrm{CPLA}_{2} \alpha$ expression also correlated with two different profile signatures that are predictive of poor prognosis: the 70 gene (van 't Veer et al, 2002) and the wound response (Chang et al, 2005) signatures (Figure 2B).

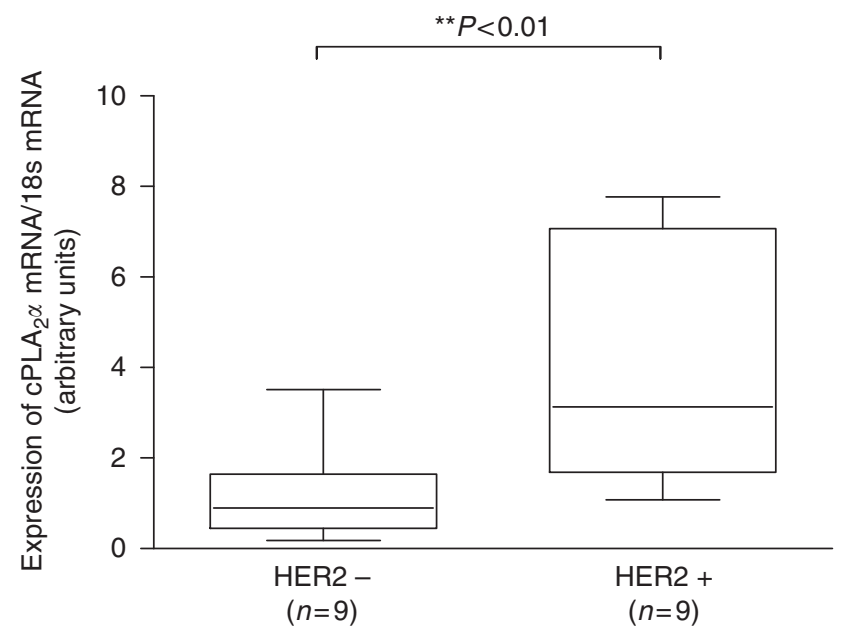

Figure I Expression of $\mathrm{CPLA} \mathrm{A}_{2} \alpha \mathrm{mRNA}$ according to HER2 expression in breast cancer tissue samples. Samples from 18 breast cancer patients were separated into HER2-positive and HER2-negative groups according to qRT-PCR quantification and threshold of HER2 expression, as indicated in Materials and methods. Expression of $c P L A_{2} \alpha$ mRNA, measured by qRT$P C R$, was compared amongst the two groups. $* * P<0.01$ measured by Student's t-test.
Increased expression of $\mathrm{cPLA}_{2} \alpha$ mRNA correlates with clinical parameters of poor prognosis in a cohort of breast cancer patients

Expression of $\mathrm{CPLA}_{2} \alpha$ was investigated in a second gene expression microarray dataset comprising 295 breast cancer patients from The Netherland Cancer Institute (van de Vijver et al, 2002). There was a positive correlation between $\mathrm{CPLA}_{2} \alpha$ and EGFR mRNA expression (Pearson $R^{2}=0.056, P<0.0001$ ), but no correlation between cPLA $_{2} \alpha$ and HER2 expression $(P=0.7816)$. High $\mathrm{cPLA}_{2} \alpha$ mRNA abundance correlated with ER-negative status as opposed to ER positive $(P<0.0001)$, and with basal phenotype compared with luminal $(P<0.0001$; Figure $3 \mathrm{~A}$ and $\mathrm{B}$ and Supplementary Table S2). When compared with the TNM staging system, high $\mathrm{CPLA}_{2} \alpha$ expression correlated with size of the primary tumour $(\mathrm{T})$ of $>2 \mathrm{~cm}(P<0.05$; Figure $3 \mathrm{C})$ but not with lymph node involvement $(\mathrm{N})$ or with distant metastasis $(\mathrm{M})$. Increased $\mathrm{CPLA}_{2} \alpha$ abundance also correlated with histology grade 3 tumours compared with grade 1 and $2(P<0.05)$ and with the activated wound response model (a gene signature predictive of poor prognosis (Chang et al, 2005)) $(P<0.001)$. Correlation with the 70 gene profile signature almost reached statistical significance $(P=0.0528$; Figure $3 \mathrm{D}-\mathrm{F}$ and Supplementary table S2). In spite of the correlation between high $\mathrm{CPLA}_{2} \alpha$ mRNA expression and different clinical parameters characteristic of poor prognosis, $\mathrm{CPLA}_{2} \alpha$ itself was not associated with poor prognosis in both univariate and multivariate analysis (Figure 4 and Supplementary Table S3). Patients with high $\mathrm{cPLA}_{2} \alpha$ expression had a reduced 7 years OS compared with patients expressing low $\mathrm{CPLA}_{2} \alpha$, but this was not statistically significant $(P=0.1$; Figure $4 \mathrm{~A})$. However, when the same analysis was repeated separately on luminal and basal tumour patient data, high $\mathrm{CPLA}_{2} \alpha$ expression was associated with reduced OS in patients with luminal cancer $(P<0.01$; Figure $4 \mathrm{~B}$ and $\mathrm{C})$.

\section{Increased expression of $\mathrm{CPLA}_{2} \alpha$ correlates with poor response to endocrine therapy in breast cancer patients}

Increased expression of EGFR/HER2 and decreased expression of $\mathrm{ER} \alpha$ are molecular markers associated with resistance to endocrine therapy in breast cancer (Pietras, 2003). We investigated the relationship between $\mathrm{CPLA}_{2} \alpha$ expression and the development of endocrine resistance in The Netherland Cancer Institute cohort

A

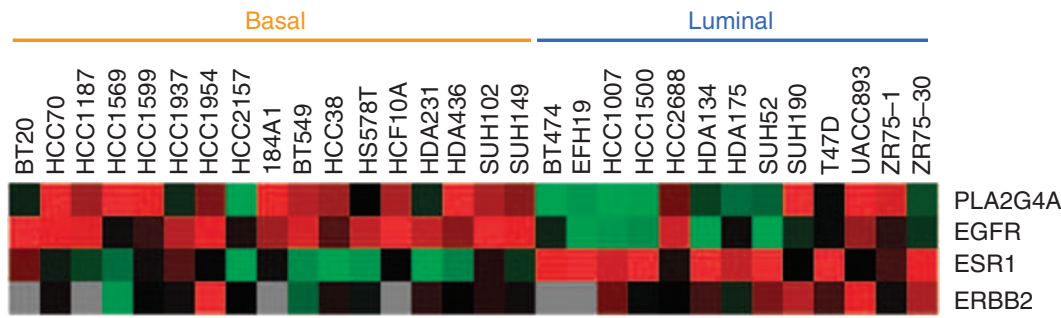

B
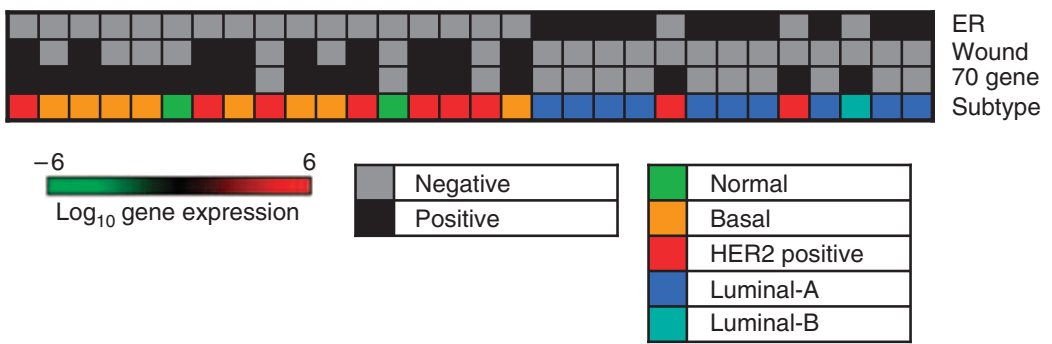

Figure 2 Correlation between cPLA $2 \alpha$ mRNA expression and clinicopathological characteristics in a panel of 30 breast cancer cell lines. (A) Log 2 expression levels of $c P L A_{2} \alpha$ (PLA2G4A), ER $\alpha$ (ESRI), EGFR and HER2 (ERBB2) are depicted by the indicated pseudo-colour scale (grey represents missing or poorly measured data). (B) Classification of cell lines by ER status, by positivity for 70 gene and wound response signatures, and by nearest resemblance to tumour gene expression subtype (normal, luminal A, luminal B, HER2 positive, basal-like). 
A
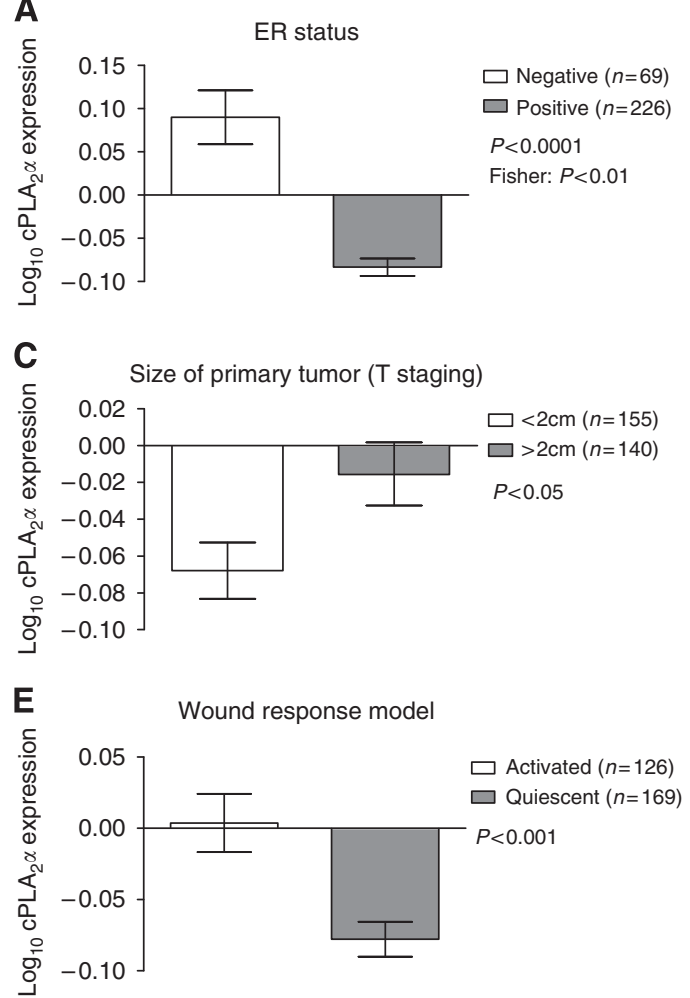

B
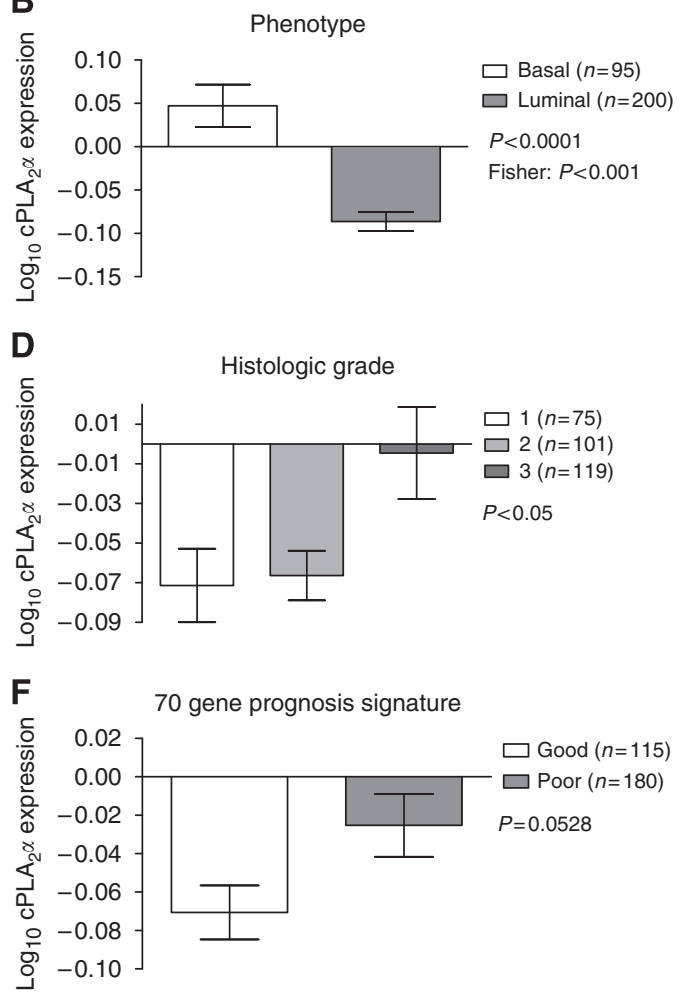

Figure 3 Correlation between $\mathrm{CPLA}_{2} \alpha$ mRNA expression and clinicopathological characteristics in 295 breast cancer patients. Log 10 expression of CPLA $2 \alpha$ compared with $(\mathbf{A})$ ER status, $(\mathbf{B})$ basal-luminal phenotype, $(\mathbf{C})$ size of primary tumour, $(\mathbf{D})$ histology grade, $(\mathbf{E})$ the wound response signature and $(\mathbf{F})$ the 70 gene signature. The number of patients $(n)$ in each group is indicated. P-values were calculated with Student's t-test, or with Fisher's exact test (where indicated).
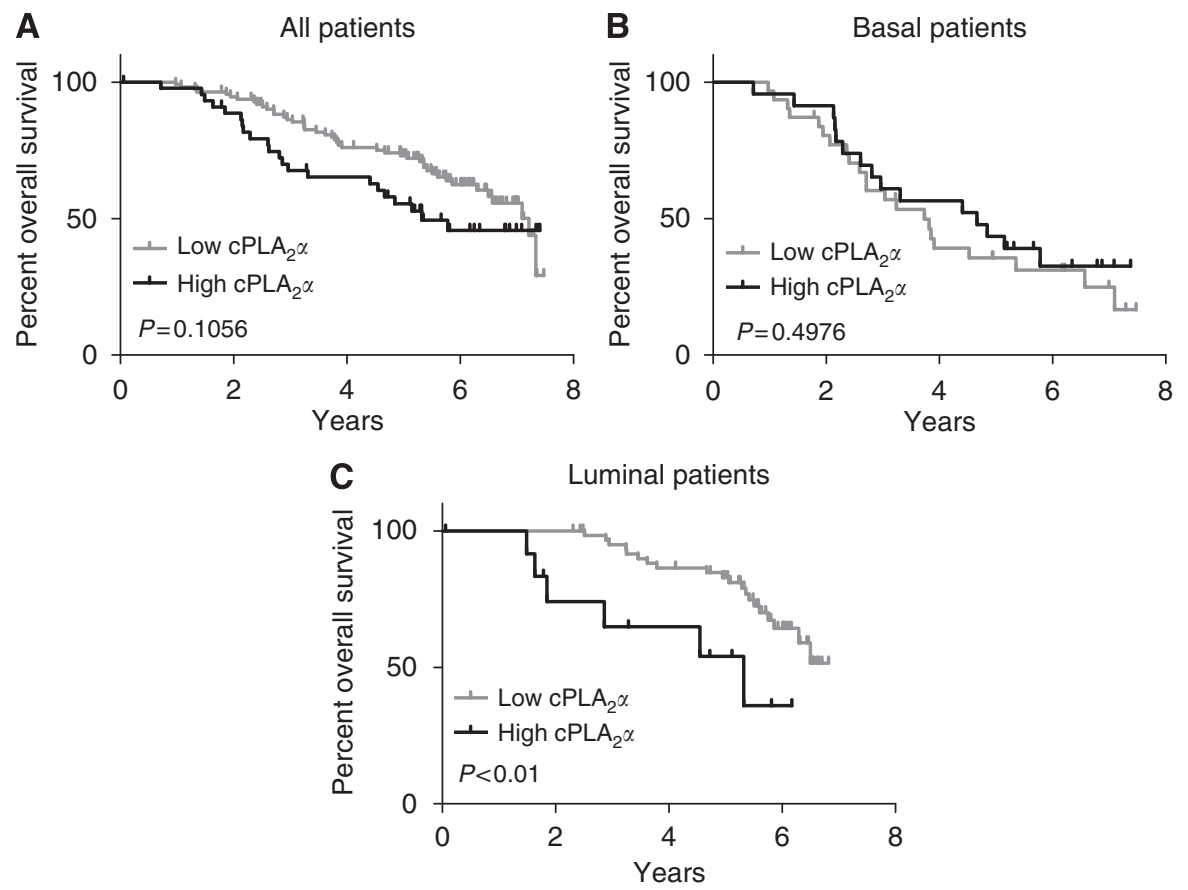

Figure 4 Prognostic value of cPLA 2 expression on the overall survival of 295 breast cancer patients. Kaplan-Meier analysis of the probability of overall survival among $(\mathbf{A})$ all 295 breast cancer patients (high $n=45$ vs low $n=112$ ), or patients with $(\mathbf{B})$ basal (high $n=23$ vs low $n=31$ ) and $(\mathbf{C})$ luminal cancers (high $n=13$ vs low $n=62$ ), according to $C P L A_{2} \alpha$ expression. Survival curves were compared with the Cox-Mantel log-rank test, and the corresponding $P$-value is indicated.

of 295 breast cancer patients (see Supplementary Table S4 for subset sizes). Although patients who expressed $\mathrm{CPLA}_{2} \alpha$ most highly, had a reduced RFS when treated with endocrine therapy compared with patients who received no adjuvant hormonal treatment, according to Kaplan - Meier univariate survival analysis, these data did not reach statistical significance $(P=0.56$ and 
$P=0.09$, respectively; Figure 5A). However, the increased RFS measured in patients treated with adjuvant hormonal therapy compared with patients only treated with local therapy (log-rank test, $P<0.05)$ was completely lost in the subset of patients expressing high levels of $\mathrm{CPLA}_{2} \alpha \quad(P=0.33)$, with the most pronounced difference between the $\mathrm{CPLA}_{2} \alpha$-positive patients and the whole cohort observed within the first 5 years after diagnosis (Figure 5B). For the whole cohort of patients, endocrine therapy increased the mean time before first metastasis by 1 year compared with receiving local therapy only $(8.52 \pm 0.45$ and $7.54 \pm 0.29$ years, respectively), and increased the number of patients free of distant metastasis 5 years after diagnosis by $18 \%$ (76.3\% with hormonal therapy $v s 58.6 \%$ with no hormonal therapy). These effects were lost in high $\mathrm{CPLA}_{2} \alpha$-expressing patients, for both the mean time before first metastasis $(7.11 \pm 0.72$ years with hormonal therapy $v s$ $7.96 \pm 0.42$ years without hormonal therapy) and the percentage of patients free of distant metastasis at 5 years after diagnosis $(64.7 \%$ with hormonal therapy $v s \quad 64.9 \%$ without hormonal therapy). A Cox proportional multivariate analysis performed on this data set (Table 1) showed that low $\mathrm{CPLA}_{2} \alpha$ mRNA expression and adjuvant hormonal therapy were independent prognostic factors, and were predictive of increased RFS in the first 5 years of patient follow-up (odds ratio: $0.244,95 \%$ CI: $0.069-0.862, P<0.05$ ). This analysis also included ER and HER2 expression as continuous variables.

\section{DISCUSSION}

The role of eicosanoid signalling in carcinogenesis has been the subject of intense investigation over the past 20 years, particularly in the context of COX-2 over-expression. The dysregulation of $\mathrm{cPLA}_{2} \alpha$, through alterations in its functional activity or in expression levels, is also a common feature of many types of human cancer including mammary adenocarcinoma, leading to high levels of proliferative eicosanoids (Nakanishi and Rosenberg, 2006; Thomas et al, 2008). Antagonism of $\mathrm{CPLA}_{2} \alpha$ would limit free intracellular arachidonic acid availability; this in turn would have a dual effect on tumour cells by reducing proliferative $\mathrm{PGE}_{2}$ production and also block the supply of arachidonic acid to alternate pathways, such as those mediated by COX-1 and lipoxygenase. This alternate metabolism of arachidonic acid represents a serious negative side effect that was reported with the clinical use of COX-2-specific inhibitors, which may be over come through specific $\mathrm{cPLA}_{2} \alpha$ antagonism (Laye and Gill, 2003). We previously demonstrated that selective pharmacological inhibition of $\mathrm{CPLA}_{2} \alpha$ leads to the growth inhibition of breast cancer cells in vitro (Caiazza et al, 2010). It is, therefore, crucial to determine whether $\mathrm{cPLA}_{2} \alpha$ has any value as a putative therapeutic target in vivo, and also to investigate which specific subset of breast cancer patients may benefit the most from such intervention. We previously found through in vitro studies that there was a correlation between high $\mathrm{CPLA}_{2} \alpha$ expression and HER2 overexpression at both mRNA and protein level in a small panel of breast cancer cell lines (Caiazza et al, 2010). This expression correlation may be explained by a bi-directional cross-talk between the two proteins; where by HER2 can regulate the expression of $\mathrm{CPLA}_{2} \alpha$ which in turn functions by modulating the transcription of HER2 through the production of $\mathrm{PGE}_{2}$ (Benoit et al, 2004; Caiazza et al, 2010). A similar synergy was also reported between HER2 and COX-2 expression (Benoit et al, 2004; Wang

Table I Multivariable proportional-hazard (cox) analysis of the risk of distant metastasis as a first event within 5 years of diagnosis

\begin{tabular}{|c|c|c|}
\hline Variable & $\begin{array}{c}\text { Hazard } \\
\text { ratio }\left(95 \% \mathrm{Cl}^{\mathrm{a}}\right)\end{array}$ & $P$-value \\
\hline Low cPLA $\alpha$ (vs high) & $0.244(0.069-0.862)$ & 0.028 \\
\hline Hormonal therapy (vs no hormonal therapy) & $0.384(0.172-0.855)$ & 0.019 \\
\hline
\end{tabular}

Abbreviations: $\mathrm{Cl}=$ confidence interval, $\quad \mathrm{CPLA} \alpha=$ cytosolic phospholipase $\mathrm{A}_{2} \alpha$ $\mathrm{ER}=$ oestrogen receptor. ${ }^{\mathrm{a}} \mathrm{Cl}$ (adjusted for ER and HER2 status). Total patients $=124$ (hormonal $=20$, no hormonal $=104$, PLA2 $\alpha$ high $=4$, PLA2 $\alpha$ low $=120$ ).
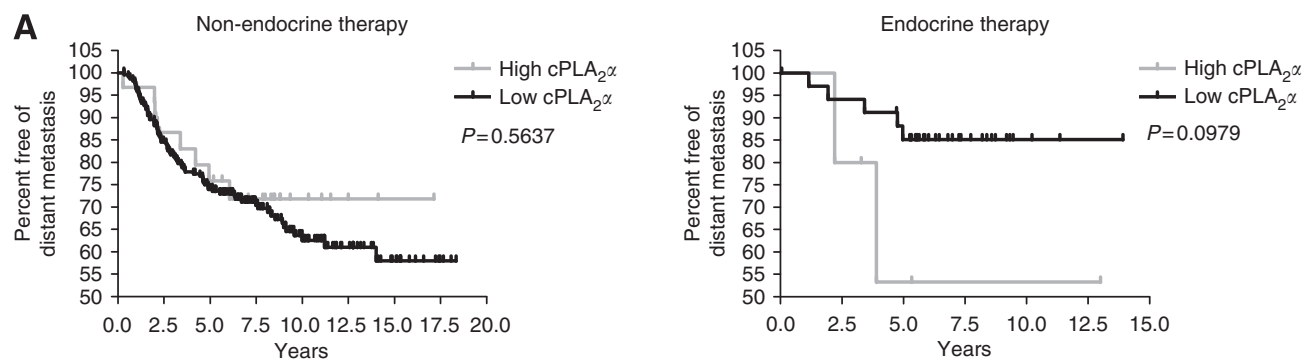

B

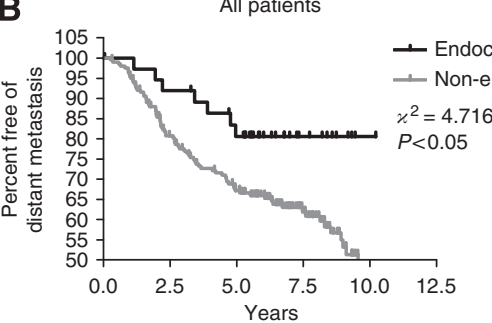

$\mathrm{CPLA}_{2} \alpha$-positive patients

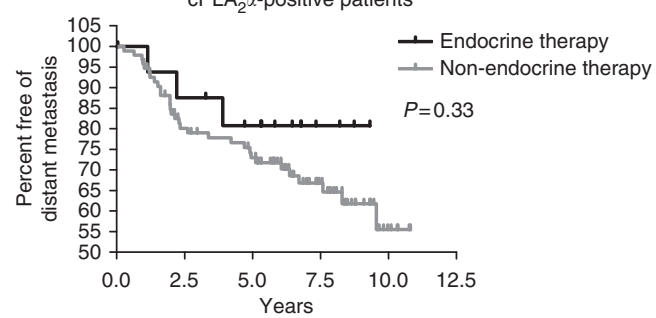

Figure 5 Role of $\mathrm{cPLA}_{2} \alpha$ in the early development of endocrine resistance in 295 breast cancer patients. Kaplan-Meier analysis of the probability that patients would remain free of distant metastases $(\mathbf{A})$ among patients treated only with radiotherapy/chemotherapy and patients treated with adjuvant tamoxifen therapy, according to cPLA $\alpha$ expression (high, $n=3 \mathrm{I}$ vs low, $n=224$ for radio/chemotherapy and high, $n=5$ vs low, $n=35$ for adjuvant therapy), total follow-up time of 17 years; (B) among all patients or patients with high cPLA 2 expression, according to the presence or absence of adjuvant endocrine therapy (non-endocrine, $n=203$ vs endocrine, $n=38$ for all patients; non-endocrine, $n=94$ vs endocrine, $n=17$ for cPLA $\alpha$ positive patients), follow-up time of 10 years; ( $\mathbf{A}$ and $\mathbf{B}$ ) Survival curves were compared using the Cox-Mantel log-rank test. The corresponding $\chi^{2}$ values and $P$-values are indicated. The threshold for $\mathrm{CPLA} 2 \alpha$ dichotomisation in $(\mathbf{B})$ was lowered to the median in order to include more patients; the statistical significance was retained as compared with the analysis with the median cutoff point (not shown), suggesting the absence of bias because of the low number of patients. 
et al, 2004), and as a consequence COX-2 over-expression is associated with HER2-driven tumourigenesis in vivo (Howe et al, 2005; Dillon et al, 2008). In this study, we report a correlation between $\mathrm{cPLA}_{2} \alpha$ and high HER2 mRNA expression in a group of 18 breast cancer patients. This was subsequently confirmed by the analysis of a gene expression microarray of 30 breast cancer cell lines, in which high mRNA expression of $\mathrm{CPLA}_{2} \alpha$ correlated with high EGFR expression and with the HER2-positive and basal-like subtypes of breast cancer in cell lines showing basal phenotype. The molecular classification of breast cancer, based on the seminal work of Perou et al, 2000 (Sorlie et al, 2001), identified five main subtypes of tumours with distinct patterns of gene expression and different disease outcomes: luminal A and luminal B cancers (mainly ER positive and sensitive to endocrine therapy), HER2 positive (ER negative or with low ER expression, HER2 over-expressing and resistant to endocrine therapy), basal-like (ER negative, PR negative and HER2 negative, also called triple negative) and normal-like. In our in silico analysis of gene expression microarrays from both cell lines and patients, elevated $\mathrm{CPLA}_{2} \alpha$ mRNA expression was associated with clinical parameters for poor prognosis, which correlate with the HER2-positive and basal-like subtypes of breast cancer. Low ER expression, high EGFR expression, activation of poor prognosis profile signatures, large tumour size and high histological grade all associated significantly with high $\mathrm{cPLA}_{2} \alpha$ mRNA expression. So supporting the hypothesis that $\mathrm{CPLA}_{2} \alpha$ could represent a specific therapeutic target for a clinically challenging subset of breast cancer patients with highly invasive, endocrine resistant tumours of the HER2positive or triple-negative subtype. In spite of the association between $\mathrm{CPLA}_{2} \alpha$ expression and different clinicopathological parameters characteristic of poor prognosis, $\mathrm{CPLA}_{2} \alpha$ was not directly associated with poor prognosis, but only correlated with reduced overall survival in patients with the luminal type of cancer. This result suggested that $\mathrm{CPLA}_{2} \alpha$ could predict prognosis specifically in luminal breast cancers, and hence be indicative of the response to endocrine therapy. Over-expression of EGFR/ HER2 and downregulation of ER are molecular markers associated with de novo and acquired resistance to endocrine therapy in both pre-clinical models of breast cancer and in clinical studies (Knowlden et al, 2003; Schiff et al, 2005). Several studies have provided indirect evidence of a role for the eicosanoid signalling pathway in the development of endocrine resistance, including the $\mathrm{PGE}_{2}$-mediated, enhanced expression of HER2 (Benoit et al, 2004) and expression of aromatase (Zhao et al, 1996). Direct evidence also supports a role for COX-2 as an independent prognostic marker for poor response to tamoxifen treatment in breast cancer patients (Dillon et al, 2008). In this study, we analysed $\mathrm{CPLA}_{2} \alpha$ expression with regards to hormonal treatment in a population of 295 breast cancer patients that were either treated with adjuvant tamoxifen (alone or in combination with chemotherapy) or did not

\section{REFERENCES}

Barberis M, Pellegrini C, Cannone M, Arizzi C, Coggi G, Bosari S (2008) Quantitative PCR and HER2 testing in breast cancer: a technical and cost-effectiveness analysis. Am J Clin Pathol 129: $563-570$

Benoit V, Relic B, Leval Xd X, Chariot A, Merville MP, Bours V (2004) Regulation of HER-2 oncogene expression by cyclooxygenase- 2 and prostaglandin E2. Oncogene 23: $1631-1635$

Caiazza F, Harvey BJ, Thomas W (2010) Cytosolic phospholipase A2 activation correlates with HER2 overexpression and mediates estrogen-dependent breast cancer cell growth. Mol Endocrinol 24: $953-968$

Chang HY, Nuyten DS, Sneddon JB, Hastie T, Tibshirani R, Sorlie T, Dai H, He YD, van't Veer LJ, Bartelink H, van de Rijn M, Brown PO, van de Vijver MJ (2005) Robustness, scalability, and integration of a wound- receive any adjuvant endocrine treatment. High $\mathrm{CPLA}_{2} \alpha$ mRNA was measured in only a small subset of the patients who received endocrine therapy, and increased $\mathrm{CPLA}_{2} \alpha$ expression in this group predicted poor response to endocrine therapy in the first 5 years of follow-up in univariate analysis. The $\mathrm{CPLA}_{2} \alpha$ was also an independent prognostic marker of poor response to endocrine therapy in a multivariate analysis that also included hormonal treatment, ER and HER2 expression to adjust for any bias because of the association of $\mathrm{CPLA}_{2} \alpha$ expression with ER negativity (which itself correlates with endocrine resistance). This analysis only takes into account treatment with tamoxifen and does not include other types of endocrine therapies, such as aromatase inhibition.

In summary, the data presented in this work provide evidence of a role for $\mathrm{CPLA}_{2} \alpha$ in breast cancer progression; $\mathrm{CPLA}_{2} \alpha$ expression correlated with clinicopathological parameters of poor prognosis and with highly aggressive tumours characterised by low ER expression, high EGFR expression and basal phenotype. This work also provides evidence to support a role for $\mathrm{CPLA}_{2} \alpha$ as a predictive marker for poor prognosis, and suggest a role in the early onset of resistance to tamoxifen therapy, in luminal cancers; this is in common with what has been previously reported for COX-2 expression (Dillon et al, 2008). Targeting eicosanoid pathway intermediates may prove a valuable strategy to overcome acquired resistance to tamoxifen treatment of breast cancer, and it has been suggested that specific antagonism of $\mathrm{CPLA}_{2} \alpha$ could be useful in augmenting treatment (Thomas et al, 2008). In this study, we show that patients with highly aggressive cancers of the HER2-positive and basal-like subtypes, as well as patients with luminal tumours who developed resistance to endocrine therapy, are most likely to benefit from such a therapeutic strategy, due to their higher $\mathrm{cPLA}_{2} \alpha$ expression levels.

\section{ACKNOWLEDGEMENTS}

We thank Dr Charles Perou and Dr Fan Cheng (University of North Carolina at Chapel Hill, USA) for their assistance with data retrieval. The bioinformatic expertise of Dr Alessandro Montaghi (University of Florence, Italy) is also acknowledged. This work was supported by the Higher Education Authority of Ireland (PRTLI Cycle 4) through the National Bio-Photonics and Imaging Platform, and by a PhD scholarship to FC from The Royal College of Surgeons in Ireland.

Supplementary Information accompanies the paper on British Journal of Cancer website (http://www.nature.com/bjc) response gene expression signature in predicting breast cancer survival. Proc Natl Acad Sci USA 102: 3738-3743

Dillon MF, Stafford AT, Kelly G, Redmond AM, Mcllroy M, Crotty TB, McDermott E, Hill AD, Young LS (2008) Cyclooxygenase-2 predicts adverse effects of tamoxifen: a possible mechanism of role for nuclear HER2 in breast cancer patients. Endocr Relat Cancer 15: $745-753$

Gown AM (2008) Current issues in ER and HER2 testing by IHC in breast cancer. Mod Pathol 21(Suppl 2): S8-S15

Hortobagyi GN, de la Garza Salazar J, Pritchard K, Amadori D, Haidinger R, Hudis CA, Khaled H, Liu MC, Martin M, Namer M, O’Shaughnessy JA, Shen ZZ, Albain KS (2005) The global breast cancer burden: variations in epidemiology and survival. Clin Breast Cancer 6: $391-401$

Howe LR (2007) Inflammation and breast cancer. Cyclooxygenase/ prostaglandin signaling and breast cancer. Breast Cancer Res 9: 210 
Howe LR, Chang SH, Tolle KC, Dillon R, Young LJ, Cardiff RD, Newman RA, Yang P, Thaler HT, Muller WJ, Hudis C, Brown AM, Hla T, Subbaramaiah K, Dannenberg AJ (2005) HER2/neu-induced mammary tumorigenesis and angiogenesis are reduced in cyclooxygenase-2 knockout mice. Cancer Res 65: 10113-10119

Hu Z, Fan C, Oh DS, Marron JS, He X, Qaqish BF, Livasy C, Carey LA, Reynolds E, Dressler L, Nobel A, Parker J, Ewend MG, Sawyer LR, Wu J, Liu Y, Nanda R, Tretiakova M, Ruiz Orrico A, Dreher D, Palazzo JP, Perreard L, Nelson E, Mone M, Hansen H, Mullins M, Quackenbush JF, Ellis MJ, Olopade OI, Bernard PS, Perou CM (2006) The molecular portraits of breast tumors are conserved across microarray platforms. BMC Genomics 7: 96

Jemal A, Ward E, Thun MJ (2007) Recent trends in breast cancer incidence rates by age and tumor characteristics among U.S. women. Breast Cancer Res 9: $\mathrm{R} 28$

Jordan VC (2007) Chemoprevention of breast cancer with selective oestrogen-receptor modulators. Nat Rev Cancer 7: 46-53

Kao J, Salari K, Bocanegra M, Choi YL, Girard L, Gandhi J, Kwei KA, Hernandez-Boussard T, Wang P, Gazdar AF, Minna JD, Pollack JR (2009) Molecular profiling of breast cancer cell lines defines relevant tumor models and provides a resource for cancer gene discovery. PLoS One 4: e6146

Knowlden JM, Hutcheson IR, Jones HE, Madden T, Gee JM, Harper ME, Barrow D, Wakeling AE, Nicholson RI (2003) Elevated levels of epidermal growth factor receptor/c-erbB2 heterodimers mediate an autocrine growth regulatory pathway in tamoxifen-resistant MCF-7 cells. Endocrinology 144: 1032 - 1044

Kulka J, Tokes AM, Kaposi-Novak P, Udvarhelyi N, Keller A, Schaff Z (2006) Detection of HER-2/neu gene amplification in breast carcinomas using quantitative real-time PCR - a comparison with immunohistochemical and FISH results. Pathol Oncol Res 12: 197-204

Laye JP, Gill JH (2003) Phospholipase A2 expression in tumours: a target for therapeutic intervention? Drug Discov Today 8: 710-716

Leslie CC (1997) Properties and regulation of cytosolic phospholipase A2. $J$ Biol Chem 272: 16709-16712

Linn SC, Van 't Veer LJ (2009) Clinical relevance of the triple-negative breast cancer concept: genetic basis and clinical utility of the concept. Eur J Cancer 45(Suppl 1): 11-26

Lopez-Tarruella S, Schiff R (2007) The dynamics of estrogen receptor status in breast cancer: re-shaping the paradigm. Clin Cancer Res 13: $6921-6925$

Nakanishi M, Rosenberg DW (2006) Roles of cPLA2alpha and arachidonic acid in cancer. Biochim Biophys Acta 1761: 1335-1343

Pavlidis P, Noble WS (2003) Matrix2png: a utility for visualizing matrix data. Bioinformatics 19: 295-296

Perou CM, Sorlie T, Eisen MB, van de Rijn M, Jeffrey SS, Rees CA, Pollack JR, Ross DT, Johnsen H, Akslen LA, Fluge O, Pergamenschikov A, Williams C, Zhu SX, Lonning PE, Borresen-Dale AL, Brown PO, Botstein D (2000) Molecular portraits of human breast tumours. Nature 406: $747-752$

Pietras RJ (2003) Interactions between estrogen and growth factor receptors in human breast cancers and the tumor-associated vasculature. Breast J 9: $361-373$
Rabindran SK (2005) Antitumor activity of HER-2 inhibitors. Cancer Lett 227: $9-23$

Ristimaki A, Sivula A, Lundin J, Lundin M, Salminen T, Haglund C, Joensuu H, Isola J (2002) Prognostic significance of elevated cyclooxygenase-2 expression in breast cancer. Cancer Res 62: 632-635

Schiff R, Massarweh SA, Shou J, Bharwani L, Arpino G, Rimawi M, Osborne CK (2005) Advanced concepts in estrogen receptor biology and breast cancer endocrine resistance: implicated role of growth factor signaling and estrogen receptor coregulators. Cancer Chemother Pharmacol 56(Suppl 1): $10-20$

Sorlie T, Perou CM, Tibshirani R, Aas T, Geisler S, Johnsen H, Hastie T, Eisen MB, van de Rijn M, Jeffrey SS, Thorsen T, Quist H, Matese JC, Brown PO, Botstein D, Eystein Lonning P, Borresen-Dale AL (2001) Gene expression patterns of breast carcinomas distinguish tumor subclasses with clinical implications. Proc Natl Acad Sci USA 98: 10869-10874

Subbaramaiah K, Norton L, Gerald W, Dannenberg AJ (2002) Cyclooxygenase-2 is overexpressed in HER-2/neu-positive breast cancer: evidence for involvement of AP-1 and PEA3. J Biol Chem 277: 18649-18657

Thomas W, Caiazza F, Harvey BJ (2008) Estrogen, phospholipase A and breast cancer. Front Biosci 13: 2604-2613

Thomas W, Coen N, Faherty S, Flatharta CO, Harvey BJ (2006) Estrogen induces phospholipase $\mathrm{A}(2)$ activation through ERK1/2 to mobilize intracellular calcium in MCF-7 cells. Steroids 71: 256-265

Vadlamudi R, Mandal M, Adam L, Steinbach G, Mendelsohn J, Kumar R (1999) Regulation of cyclooxygenase-2 pathway by HER2 receptor. Oncogene 18: 305 -314

van 't Veer LJ, Dai H, van de Vijver MJ, He YD, Hart AA, Mao M, Peterse HL, van der Kooy K, Marton MJ, Witteveen AT, Schreiber GJ, Kerkhoven RM, Roberts C, Linsley PS, Bernards R, Friend SH (2002) Gene expression profiling predicts clinical outcome of breast cancer. Nature 415: 530-536

van de Vijver MJ, He YD, van't Veer LJ, Dai H, Hart AA, Voskuil DW, Schreiber GJ, Peterse JL, Roberts C, Marton MJ, Parrish M, Atsma D, Witteveen A, Glas A, Delahaye L, van der Velde T, Bartelink H, Rodenhuis S, Rutgers ET, Friend SH, Bernards R (2002) A geneexpression signature as a predictor of survival in breast cancer. $N$ Engl $J$ Med 347: 1999-2009

Vinatzer U, Dampier B, Streubel B, Pacher M, Seewald MJ, Stratowa C, Kaserer $\mathrm{K}$, Schreiber M (2005) Expression of HER2 and the coamplified genes GRB7 and MLN64 in human breast cancer: quantitative real-time reverse transcription-PCR as a diagnostic alternative to immunohistochemistry and fluorescence in situ hybridization. Clin Cancer Res 11: 8348-8357

Wang SC, Lien HC, Xia W, Chen IF, Lo HW, Wang Z, Ali-Seyed M, Lee DF, Bartholomeusz G, Ou-Yang F, Giri DK, Hung MC (2004) Binding at and transactivation of the COX-2 promoter by nuclear tyrosine kinase receptor ErbB-2. Cancer Cell 6: 251-261

Wulfing P, Diallo R, Muller C, Wulfing C, Poremba C, Heinecke A, Rody A, Greb RR, Bocker W, Kiesel L (2003) Analysis of cyclooxygenase-2 expression in human breast cancer: high throughput tissue microarray analysis. J Cancer Res Clin Oncol 129: 375-382

Zhao Y, Agarwal VR, Mendelson CR, Simpson ER (1996) Estrogen biosynthesis proximal to a breast tumor is stimulated by PGE2 via cyclic AMP, leading to activation of promoter II of the CYP19 (aromatase) gene. Endocrinology 137: 5739-5742 